\title{
The Common Coordinate Framework (CCF) Organ VR Gallery
}

\author{
Andreas Bueckle ${ }^{1}$ \\ Department of Intelligent \\ Systems Engineering, \\ Luddy School of \\ Informatics, Computing, \\ and Engineering, Indiana \\ University, Bloomington, \\ Indiana, USA
}

\author{
Kristen M. Browne ${ }^{2}$ \\ Bioinformatics and \\ Computational Biosciences \\ Branch, Office of Cyber \\ Infrastructure and \\ Computational Biology, \\ National Institute of Allergy \\ and Infectious Diseases, \\ National Institutes of \\ Health, Bethesda, \\ Maryland, USA
}

\author{
Bruce W. Herr II \\ Department of Intelligent \\ Systems Engineering, \\ Luddy School of \\ Informatics, Computing, \\ and Engineering, Indiana \\ University, Bloomington, \\ Indiana, USA
}

\author{
Katy Börner ${ }^{4}$ \\ Department of Intelligent \\ Systems Engineering, \\ Luddy School of \\ Informatics, Computing, \\ and Engineering, Indiana \\ University, Bloomington, \\ Indiana, USA
}

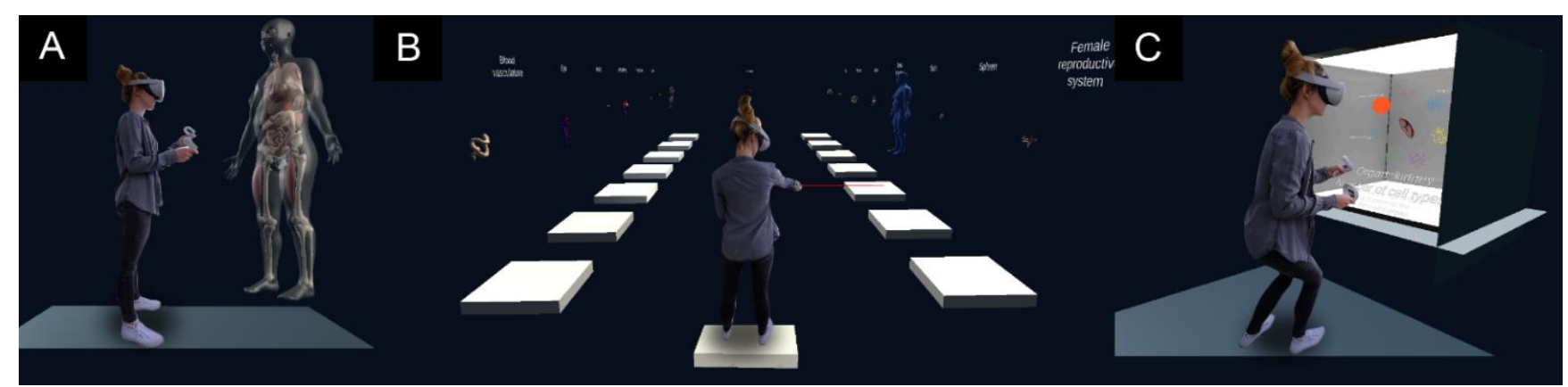

Figure 1: Three stages. A: the whole body is visible. B: a gallery of individual organs. C: a user explores cells around a kidney in a virtual box.

\section{ABstract}

The CCF Organ VR Gallery lets the user explore 21 human organs, journeying from the Whole Body, to the Organ, to the Cell stage and back, presented in real-world size and 3D. The user discovers hidden regions by exploding and collapsing organs into their individual anatomical structures. We show cell type populations for a kidney using a data-driven dot density visualization. The organ models were developed to map trillions of cells for the Human BioMolecular Atlas Program (HuBMAP).

Keywords: Virtual reality, mesh geometry models, interactive learning environments, bioinformatics.

Index Terms: [Human-centered computing]:human computer interaction (HCI)-interaction paradigms - virtual reality; [Computing methodologies]: Computer graphics-Shape modeling-Mesh geometry models; [Applied computing]: Education-Interactive learning environments; [Applied computing]: Life and medical sciences-Bioinformatics

\section{INTRODUCTION}

The human body consists of trillions of cells that work together in a harmony that has thus far defied comprehension. Even with the

\footnotetext{
1 abueckle@iu.edu

2 kristen.browne@nih.gov

3 bherr@indiana.edu

${ }^{4}$ katy@indiana.edu
}

progress in single-cell biology, bioinformatics, and microscopy, general audiences have little to no understanding of the relationship between the whole body, whole organs, organ tissue, and cells. Additionally, the three-dimensional nature of the human anatomy is hard to grasp, even without considering the complex function generated by single-cell dynamics. HuBMAP, funded by the National Institutes of Health (NIH), is an international, interdisciplinary effort to create a single-cell Human Reference Atlas (HRA) of the healthy, adult human body [7]. In order to build the HRA, massive amounts of data from a diverse set of donors is required. This data comes in the form of tissue blocks, which need to be spatially registered into representative reference organs. This is done via the Registration User Interface (RUI) [1]. Most of the 22 currently available reference organs for the RUI were created based on the Visible Human datasets [8] and are available via the CCF 3D Reference Object Library [3]. (Note that we omitted the high-polygon brain from the CCF Organ VR Gallery for performance reasons, which is why we only mention 21 organs in the accompanying video demo.) Included are sex-specific organs such as ovaries, uterus, and fallopian tubes, but also heart, kidneys, and lung. While these organs were modeled with great attention to detail using imaging and 3D software, they are typically only ever used on web browsers for tissue registration [6] and exploration [5]. VR offers a unique opportunity to experience the CCF reference organs in their anatomical and spatial context, allowing users to see hidden parts and cell type populations via our CCF Organ VR Gallery, available on GitHub: https://github.com/cns-iu/ccf-organvr-gallery 


\section{Method}

The CCF Organ VR Gallery was developed using Unity 2021 and is optimized for the Oculus Quest 2. The user explores the HRA body in three stages: Whole Body (starting stage), Organs, and Cells. The user can switch between stages at will via a level selector. In all three stages, the user can teleport with their right controller.

\subsection{Stage 1: Whole Body}

In this stage, see Figure 1A, the user explores the HRA body in real-world size and at full mesh resolution. It lets the user see position, size, rotation of all organs and their spatial context. The body can be rotated around its y-axis. The skin is transparent to offer a full view of the nested organs inside.

\subsection{Stage 2: Organs}

Once arriving in this stage, see Figure 1B, the user explores each organ separately. They are laid out over teleportation platforms with the organ name written above. By pointing their right controller at an organ and using the joystick under their thumb, the user can explode and collapse the organ into all its anatomical structures, disclosing typically hidden parts. Figure 2 shows the user pointing at the collapsed heart (Figure 2A) before exploding it (Figure 2B).

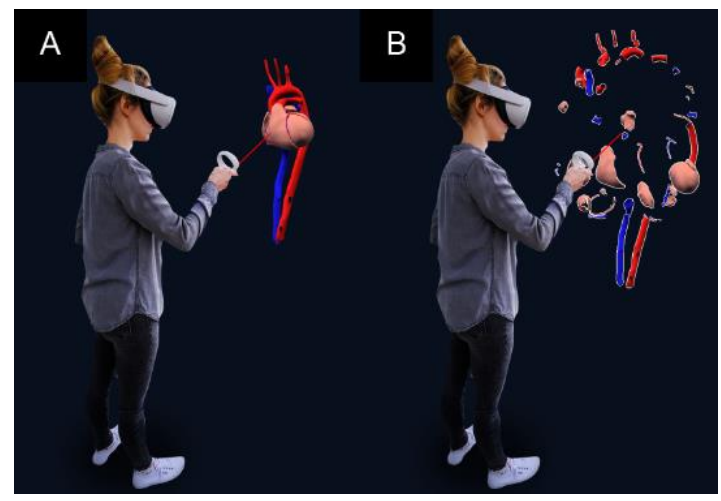

Figure 2: The user with the collapsed (A) and exploded (B) 3D model of the heart.

Note that the Organ stage only shows 15 organs. We omitted very small and hierarchically shallow $3 \mathrm{D}$ models such as the lymph node as they contain no underlying anatomical structures, making the exploding./collapsing mechanic useless for them. Also, we unified the fallopian tube, ovary, and uterus into one larger organ: the female reproductive system.

\subsection{Stage 3: Cells}

Finally, in this stage, see Figure 1C, the user explores a dot density map encoding cell type populations for the kidney. The main take-away is to understand that there are vast differences in cell counts. For example, the cell type with the highest count (epithelial cells, orange) outnumbers the cell type with the secondhighest count (B cells, yellow) at a ratio of 24:1. The data for the dot density map came from the Chan Zuckerberg Initiative's cellxgene Explorer [4].

\section{Conclusion}

The CCF reference organs is a constantly growing collection. Using other tools developed by HuBMAP, such as Anatomical Structures and Cell Types Plus Biomarkers (ASCT+B) Tables [2], they are a fundamental building block of generating the HRA by allowing to register data from incredibly diverse donors into one spatial and semantic framework. While HuBMAP as a funded research endeavor will one day be completed, part of its legacy could be educating the general audience about single-cell biology and the relationship between cells, tissue, organs, and disease. Our application serves as a vital first step in making HuBMAP and the HRA approachable, explorable, and fun. We acknowledge framerate issues due to the transparency of the skin in the Whole Body stage as well as the need for an extended tutorial on how to use the teleport and explode/collapse features. Plans for future work thus include adding animated tooltips, implementing voice-over narration, and adding more reference organs as they are released. Additionally, we hope to add visualizations of cell type populations for all organs in the Cells stage. Finally, we aim to deploy a stable version of the application to museums, schools, conferences, and other informal learning environments or areas where non-experts could be exposed to it. We hope that this effort supports the spread of the results of HuBMAP to the world.

\section{ACKNOWLEDGMENTS}

This research has been funded by the NIH under HuBMAP award OT2OD026671 [AB, BWH, KB]; by the Cellular Senescence Network (SenNet) Consortium Organization and Data Coordinating Center (CODCC) award 1U24CA268108-01 [AB, $\mathrm{BWH}, \mathrm{KB}]$; by the National Institute of Diabetes and Digestive and Kidney Diseases (NIDDK) Kidney Precision Medicine Project grant U2CDK114886 [KB]; by the Common Fund Data Ecosystem (CFDE) award OTA 20-005 OT2 OD030545 [KB]; and the National Institute of Allergy and Infectious Diseases (NIAID), Department of Health and Human Services under BCBB Support Services Contract HHSN316201300006W/HHSN27200002 [KMB]. The funders had no role in study design, data collection and analysis, decision to publish, or preparation of the manuscript. The views and conclusions contained in this document are those of the authors and should not be interpreted as representing the official policies, either expressed or implied, of the NIH.

\section{References}

[1] K. Börner et al. "Tissue Registration and Exploration User Interfaces in support of a Human Reference Atlas." https://www.biorxiv.org/content/10.1101/2021.12.30.474265 (accessed Jan 7, 2022).

[2] K. Börner et al., "Anatomical structures, cell types and biomarkers of the Human Reference Atlas," Nature Cell Biology, vol. 23, no. 11, pp. 1117-1128, 2021/11/01 2021, doi: 10.1038/s41556-021-00788-6.

[3] K. Browne et al. "HuBMAP CCF 3D Reference Object Library." https://hubmapconsortium.github.io/ccf/pages/ccf-3d-referencelibrary.html (accessed Jan 7, 2022).

[4] Chan Zuckerberg Initiative. "cellxgene." https://data.humancellatlas.org/analyze/portals/cellxgene (accessed Jan 7, 2022).

[5] Cyberinfrastructure for Network Science Center. "Exploration User Interface." https://portal.hubmapconsortium.org/ccf-eui (accessed Jan 7, 2022).

[6] Cyberinfrastructure for Network Science Center. "Registration User Interface." https://hubmapconsortium.github.io/ccf-ui/rui/ (accessed Jan 7, 2022)

[7] M. P. Snyder et al., "The human body at cellular resolution: the NIH Human Biomolecular Atlas Program," Nature, vol. 574, no. 7777, pp. 187-192, 2019/10/01 2019, doi: 10.1038/s41586-019-1629-x.

[8] V. Spitzer, M. J. Ackerman, A. L. Scherzinger, and D. Whitlock, "The visible human male: a technical report," (in eng), Journal of the American Medical Informatics Association, vol. 3, no. 2, pp. 118130, Mar-Apr 1996, doi: 10.1136/jamia.1996.96236280. 